\title{
Brain of Materials - die Plattform für Designer, Entwickler und Materialhersteller
}

Hans Peter Schlegelmilch

Brain of Materials ist eine Plattform für innovative und smarte Materialien, die Designern, Entwicklern und Ingenieuren diverser Branchen dabei hilft, ihre Produktentstehungsprozesse zu optimieren und zu beschleunigen.

Keywords: Design Research, Prozessoptimierung,

Digitale Transformation, Materialentwicklung, Plattform

\section{Digitale Transformation des Design Research Prozesses}

Bisher musste man regelmäßig Showrooms besuchen, um Materialien zu erleben. Brain of Materials überwindet die Grenzen der physischen Möglichkeiten, so dass Nutzer weltweit sofort Zugriff auf alle relevanten Informationen über Materialien haben. Mit einer Fülle an Daten werden Materialien in sehr detaillierter Form dargestellt. Insbesondere technische Eigenschaften, wie zum Beispiel Angaben zu Lichtechtheit, Entflammbarkeit, Abriebverhalten, dem Biegeverhalten eines Materials oder auch Materialdaten für strukturmechanische Berechnungen werden aufgeführt.

Das System unterstützt Designer, Ingenieure und Architekten bei der Prozessoptimierung. Übersichtlich und mit Bildern werden Werkstoffe mit verschiedenen Kategorien und Attributen gekennzeichnet. Über einen „digitalen Musterkoffer" können User eigene Kollektionen zusammenstellen, einzelne Materialkarten downloaden und auch 
ihre Kollektionen mit Anderen teilen. Brain of Materials beinhaltet neben den Beschreibungen der Eigenschaften weitere Kennzahlen, wie hochauflösende Fotos, hochwertige 3D-Scans und zertifizierte Prüfergebnisse. Ist der gewünschte Test noch nicht vorhanden, kann die Prüfung direkt über die Plattform bei einem zertifizierten Dienstleister beauftragt werden.

\section{Prüf- und Rechercheprozesse werden deutlich verkürzt}

Mit der Bereitstellung entsprechender Prüfergebnisse können Produktentwicklungszyklen erheblich beschleunigt werden - sämtliche relevanten Daten sind sofort bei der Materialrecherche verfügbar. Bisher waren spezifische Materialdaten und technische Kennzahlen nicht in der Tiefe in bestehenden Plattformen zu finden. Brain of Materials verbindet dies und bringt die Akteure zusammen.

\section{Relevante Prüfergebnisse beauftragen und eine standardisierte Darstellung für alle Materialien}

Einer der Keypoints für Materialhersteller und User, die regelmäßig mit Materialien arbeiten, ist die Beschaffung von relevanten Materialdaten. In der Regel werden Prüfdaten für Materialien von unabhängigen Laboren erstellt und von Labor zu Labor, aber auch von Prüfung zu Prüfung unterscheiden sich die erstellten Prüfberichte, die im Nachgang an die Kunden versendet werden. Diese Unterschiede machen ein schnelles Bearbeiten von Ergebnissen immer sehr schwierig und undurchsichtig. Oftmals wollen Materialhersteller ihre Materialien abprüfen lassen, aber wissen nicht genau, welche Labore bieten relevante Prüfungen an und sind diese Labore auch zertifiziert.

Genau hier setzt Brain of Materials an. Es geht nicht nur um die reine Darstellung von Daten und die Unterstützung durch hochauflösende Bilder. Über eine Order-Test Funktion können diverse Prüfungen, wie zum Beispiel Emissions-, Fogging- oder Belichtungsprüfungen beauftragt werden. Hier arbeitet Brain of Materials mit unabhängigen und von der Industrie zertifizierten Laboren zusammen. Ist für den Kunden ein passender Test nicht aufgeführt, nutzt Brain of Materials sein Netzwerk, um diese Prüfungen zusätzlich anzubieten.

Wurde die Prüfung beauftragt und durch ein zertifiziertes Labor durchgeführt, erhält der User den Testbericht in einem standardisierten Format direkt über die Plattform. Die Ergebnisse stehen zum Download bereit, können direkt auf der Materialdetailseite veröffentlicht und mit allen Usern weltweit geteilt werden.

Brain of Materials hilft nicht nur bei der Darstellung der Materialien, sondern auch bei der Weiterqualifizierung. 
Die Plattform bringt sowohl Materialien für die unterschiedlichsten Industriezweige zusammen und skaliert das Geflecht von ungeordneten technischen Datensätzen in ein geregeltes Bild, das sowohl für Laien als auch Fachpersonal alle notwendigen Daten anschaulich abbildet.

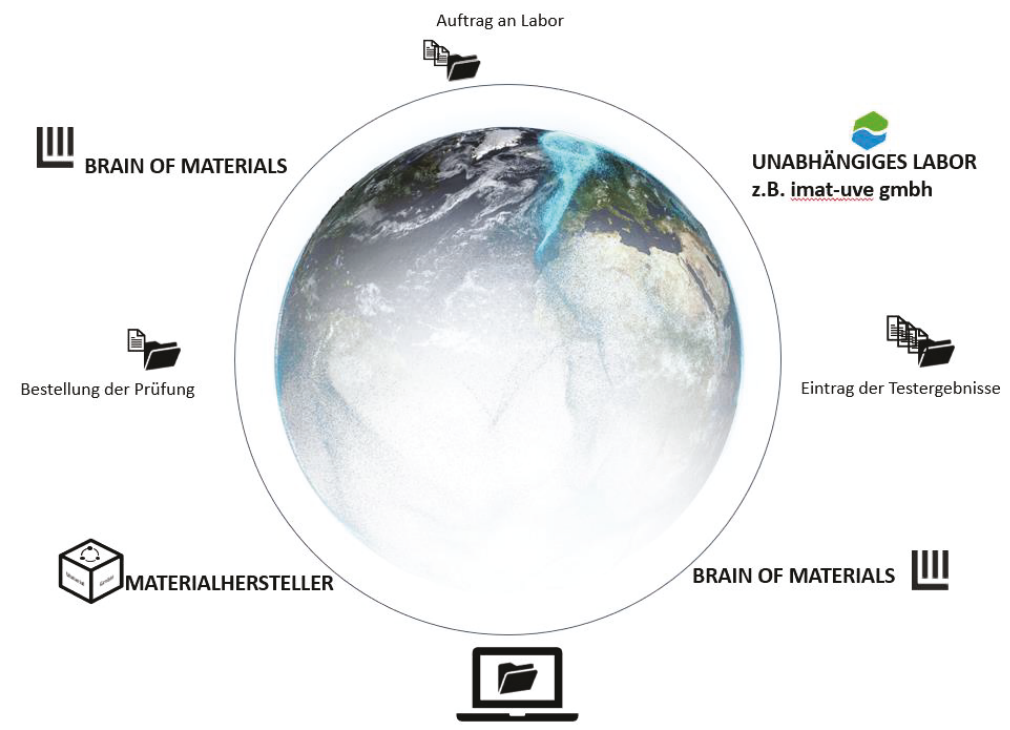

Abbildung 4: Prozess "Order Test" Brain of Materials
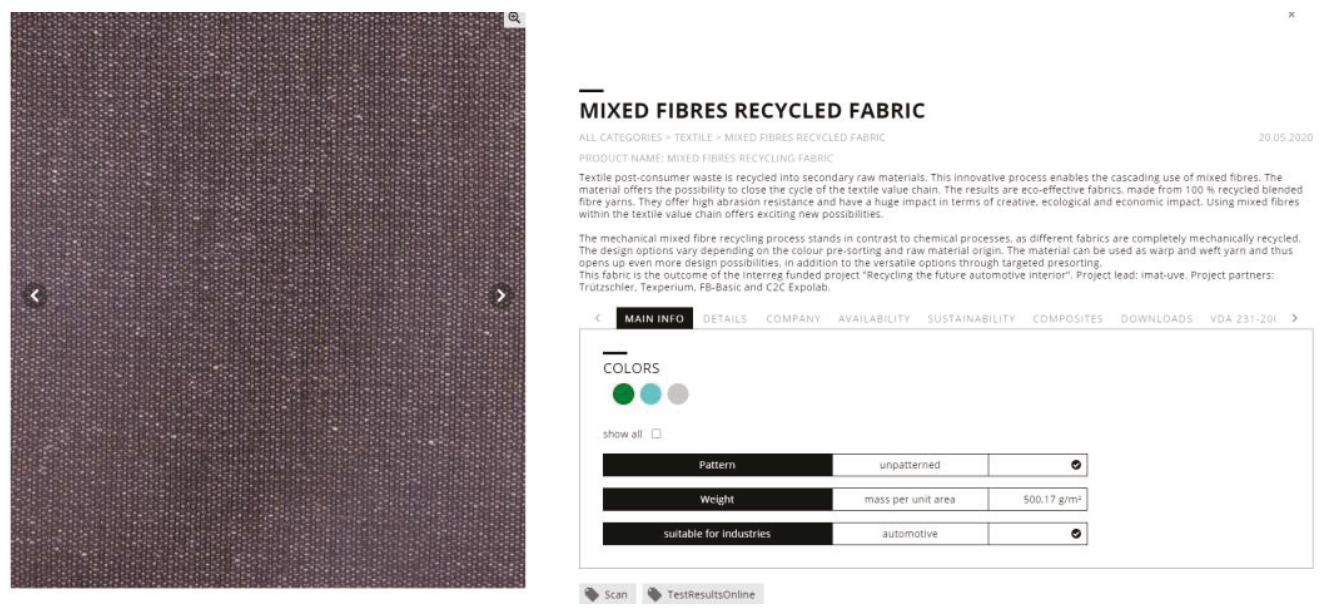

Abbildung 2: Beispiel Materialdetailseite 


\section{Kontakt}

Dr. Hans Peter Schlegelmilch

CEO \& Founder

Brain of Materials AG

Schwalmstraße 301, 41238 Mönchengladbach

hanspeter.schlegelmilch@brainofmaterials.com

www.brainofmaterials.com 